\title{
Estimation of Queue Length at Signalized Intersection : A Review
}

\author{
Kaustubh Juare ${ }^{[1]}$, Prakash Patil ${ }^{[2]}$ \\ M. Tech, Transportation Engineering, G H Raisoni College of Engineering, Nagpur, Maharashtra, India ${ }^{1}$ \\ Assistant Professor, Civil Engineering, G H Raisoni College of Engineering, Nagpur, Maharashtra, India ${ }^{2}$
}

\section{Article Info \\ Volume 8, Issue 2 \\ Page Number : 549-552}

\section{Publication Issue}

March-April-2021

\section{Article History}

Accepted : 20 April 2021

Published : 25 April 2021

\section{ABSTRACT}

In this growing world, traffic conjunction is considered as most growing problems in both urban as well as sub-urban areas. Traffic congestion is a major issue in day to day life. It has a tremendous impact on the nation's economy, air pollution, water pollution, travel behavior, accidental risk, land-use and to its end users. Various studies have shown that there is a sudden increase in the traffic congestion. Traffic congestion leads to queuing. Queuing process takes place in both signalized as well as non-signalized intersections, freeways, bottlenecks, incident sites, toll plazas, parking and the areas near the freeways. Queuing also occurs in many non transportation fields. Hence it is very important to study the queue length pattern, its estimation, methods of calculating queue length and give a solution. Hence various methods for estimation of queue length is reviewed.

Keywords : RFID (Radio Frequency Identification), Tailed Interval-based Method (TIM), Passing Time-based Method (PTM), Trail Interval and Passing Time-based Method (T-PM), MAPE (Mean Average Percentage Error).

\section{INTRODUCTION}

Traffic Congestion is a term which is characterized by longer trips, slower speeds and increase in the queue length. Intersection delays increase with the increase in the queue length. Mostly it reduces the capacity of the intersection through spillback and storage blocking between the lanes. Therefore queue length has been identified as the most important major for the calculation of operational performance of signalized intersection. Queue length is also used to determine the spacing between the intersections so that the queue does not spillover the upstream intersection. There are many methods for the estimation of queue length. We are going to study different methods to check which one is better.

\section{LITERATURE REVIEW}

[1] Dongfang Ma, Dianhai Wang, Yiming Bie, Feng Sun, and Sheng Jin-Using the data of roll time occupancy, a method was proposed to estimate the queue length in urban street networks. The main idea of this paper is that this speed of the following vehicles to pass through the detector will become smaller as the queue length in front of the queue detector becomes larger, leading to higher density with constant traffic frequency. Twelve different 
conditions were planned, and traffic data was collected using VISSIM simulation under different conditions, taking into account the relationship between roll time occupancy and queue length influenced by multiple variables such as connection length, lane width, lane number and bus ratio. Based on the simulation data review, it was decided to build an S-type logistic model for the relationship between queue lengths and roll time occupancy, and in the twelve simulation situations the fitting equations were obtained. Successive multiple linear regression with the fitting equation parameter and simulation parameter provided the average model for the relationship between queue length and roll time occupancy, and the estimation model for queue length was presented by evaluating the average relationship model equation..

[2]Aoxiang Wu, Xiaoguang Yang-Basic method for input and output approach for the queue length estimation method can only accommodate queues shorter than the distance between the vehicles detector and intersection stop line, since once the detector is filled by the queue, the total vehicle count for the arrival traffic is not available. The paper proposes a model to detect the real time queue length based on RFID (Radio frequency Identification) detector data on the basis of study of the traffic flow shock wave profile on the approach of signalized intersection. The model addresses the issue of measuring the intersection queue length by exploiting the queue length of every single vehicle instead of counting the traffic flow rate at signalized intersections. Using RFID detector at the field, the data was carried out at the Nanjing Intersection.

\section{[3] Haijian Li, Na Chen, Lingqiao Qin, Limin Jia, Jian}

Rong-The queue length estimation mechanics were modeled and analyzed by various layout strategies in this paper. Depending on the intersection percentage of the roads, several feasible techniques for magnetic sensors are proposed for intersection. In addition, a one single magnetic sensor is suggested for the queue length estimation. Specifically, three methods of single-sensor based estimation method are used which are Tail Interval-based Method (TIM), Passing Time-based Method (PTM) and Trail Interval and Passing Time-based Method (T-PM). The algorithm and the corresponding optimal layout strategy of the queue length estimation were presented on the basis of field data. The finding suggested that in terms of accuracy and robustness, T-PM had better results. It was checked that this strategy with a single magnetic sensor was the most economical strategy for estimating the queue length of the immediate past signal cycle..

\section{[4]Shuzhi Zhao, Shidong Liang, Huasheng Liu, and} Minghui Ma-Traditional methods seems insufficient to predict the length of the queue when the state of traffic varies significantly. The problem is solved in this paper by using Cell Transmission Model, a macroscopic flow of traffic, to explain the accumulation and discharge phase of vehicles at a signalized intersection. Some of its rules have been modified accordingly to adapt the model correctly to the urban traffic. Besides, in a short interval we can approximate the density of each cell of the path. Second, we define the cell were the queues tail is located. Then we determine the exact position at the back of the queue. The models are estimated by comparing the maximum queue length and average queue length predicated with the results of simulation carried out by data acquired from the field and queue tail trajectory testing.

[5]Qing CAI, Zhongyu Wang, Bing $\mathrm{Wu}, \mathrm{PhD}$ Measuring service level such as queue length at intersections in real time has received considerable attention with the explosion of traffic sensing data. In this paper, by using the data of an upstream point sensor and the moving trajectory of prob. vehicle observed by a mobile sensor, we study how to estimate real time cycle-by-cycle queue length at signalized intersections. Based on the space relationship between the rear of the queue, the point 
sensor position and the vehicle stop or start-up point, three cases may be divided. Critical break point can be identified by fusing the point data and mobile sensors to show many main stages of queue forming and dissipation. For the three cases, estimation models of the full queue length are suggested based on the Light-Witham-Richards theorem. In this paper, the algorithm has zero restriction on the traffic state (i.e. oversaturated and under-saturated conditions). In Shanghai, China, the technique is applied to the data which has been observed from the field. As a result, $11.60 \%$ and $9.98 \%$ are the mean average percentage errors (MAPEs) of the first two cases, which mean that the models have fair accuracy of estimation for the cases. For the third case, the Mean Average Percentage Error (MAPE) of the model is 26.40\%, suggesting that the output degrades but still has a reasonable precision.

[6]Anusha S. P, Lelitha Devi, Anuj Sharma-In this paper a simple method is developed for estimation of queue length at signalized intersection. Two intersections were taken in which loop detectors are installed at both entry and exit at the intersection. They are placed at $90 \mathrm{~m} \& 100 \mathrm{~m}$ behind stop line and in front of stop line respectively. The results we get is in the form of 0-1 digital pulses along with time stamps. The number of vehicles estimated are at 10 seconds time interval with the help of the advance and stop bar detectors.

[7]Bing li, Wei Cheng, and Lishan-In this paper LWR shockwave theory and Robertson's method is used to determine the real time prediction model of lane based queue length at intersections. Various assumptions are made in this model. The predicted model is used for the design of the predictive signal control.

[8]Fuliang Li, Keshuang Tang, Jiarong Yao-There are many methods to estimate the queue length based on various types of data sources. With the increase in the use of probe vehicles there is an increase in the research of estimation of cycle by cycle queue length by using probe data. In this paper they have proposed cycle by cycle queue length by using vehicle trajectory data with assumptions. With the help of the probe vehicles we can have vehicles incoming and outgoing data for both saturated and unsaturated traffic conditions.

[9] Ning $\mathrm{Wu}$ - At various signalized intersections the traffic at red end is of much importance for determining the lengths of the lane also green end is not that important but it helps in determining the queue length. In this paper the $95^{\text {th }} \& 99^{\text {th }}$ percentile od queue length is estimated under various traffic conditions . Regression model is used and then the data required for regressions is calculated by Markov Chains.

\section{[10] Zahra Amini, Ramtin Pedarsani, Alexander} Skabardonis, Pravin Varaiya-In this paper the real time queue length is estimated by using the magnetic sensors. Three types of sensors detectors are used viz. advance, stop bar, departure. The readings are recorded as per the vehicles crossings the detectors it can't give accurate results. Advance detectors are placed at upstream of the intersection at 200-300 feet and stop bar detectors are in front of the detectors and vehicle detector to detect a vehicle as it enters in the intersection.

\section{DISCUSSION}

1. Study of estimation of queue length mostly depends on three parameters viz. Detection, Estimation \& Prediction.

2. In Detection, cameras are mostly used for estimating queue at signalized intersection, main disadvantage is we cannot obtain maximum queue also it does not consider the fluctuations in the traffic flow.

3. Estimation is done by using Input-output models \& Shockwave models, as it provides better analysis framework for queue length estimation. 
4. Prediction is the new developing trend in which we can predict the traffic behavior and plan accordingly for signal priority and queue length management.

5. Manual calculation of Queue length is most accurate and reliable.

\section{REFERENCES}

[1]. Method for Queue Length Estimation in an Urban Street Network Based on Roll Time Occupancy Data. Dongfang Ma, Dianhai Wang,1 Yiming Bie,2 Feng Sun- 2012

[2]. Real-time Queue Length Estimation of Signalized Intersections Based on RFID Data. Aoxiang $\mathrm{Wu}$, Xiaoguang Yang. Conference Paper in Procedia - Social and Behavioral Sciences · June 2013

[3]. Queue length estimation at signalized intersections based on magnetic sensors by different layout strategies. Haijian $\mathrm{Li}$ a,b, $\mathrm{Na}$ Chen c, Lingqiao Qin b , Limin Jia c , Jian Rong a .World Conference on Transport Research - WCTR 2016 Shanghai.

[4]. CTM Based Real-Time Queue Length Estimation at Signalized Intersection. Shuzhi Zhao, Shidong Liang, Huasheng Liu, and Minghui Ma Oct 2015

[5]. A Shockwave Approach to Estimating Queue Length at Signalized Intersections by Fusing Data of Point and Mobile Sensors. Qing Cai, Zhongyu Wang, Bing Wu, Ph.D Submitted for presentation at 93rd annual meeting of the Transportation Research Board January 46 2014, Washington D.C.

[6]. A Method for Estimation of Queue Length S. P. Anusha Indian Institute of Technology, Madras Lelitha Devi Vanajakshi Inidan Institute of Technology, Madras Anuj Sharma University of Nebraska - Lincoln.

[7]. Real-Time Prediction of Lane-Based Queuen Lengths for Signalized Intersections Bing
Li,1,2 Wei Cheng ,1 and Lishan Li3 .Hindawi Journal of Advanced Transportation Volume 2018, Article ID 5020518

[8]. Real time queue length estimation by using vehicle trajectory data, Fuliang Li, Keshuang Tang, Jiarong Yao, Published January 1, 2017.

[9]. Estimation of queue lengths and their percentiles by Ning $\mathrm{Wu}$, Proceedings of the third international symposium on highway capacity, Copenhagen 1998.

[10]. Queue-Length Estimation Using Real-Time Traffic Data Zahra Amini, Ramtin Pedarsani, Alexander Skabardonis, Pravin Varaiya.

\section{Cite this article as :}

Kaustubh Juare, Prakash Patil, "Estimation of Queue Length at Signalized Intersection : A Review", International Journal of Scientific Research in Science and Technology (IJSRST), Online ISSN : 2395-602X, Print ISSN : 2395-6011, Volume 8 Issue 2, pp. 549-552, March-April 2021. Available at doi : https://doi.org/10.32628/IJSRST218294 Journal URL : https://ijsrst.com/IJSRST218294 